\section{Disgust - the forgotten emotion of psychiatry}

\author{
M. L. PHILLIPS, C. SENIOR, T. FAHY and A. S. DAVID
}

If asked to name common emotions, most people, including those interested in psychiatric topics, would start with happiness, sadness, and go on to fear and anger. Few would consider the emotion of disgust. We will argue that this basic emotion is important not only to many aspects of our daily lives but is also central to a range of psychiatric phenomena.

\section{EMOTIONS}

What are emotions? Dualist, or 'feeling', theories proposed by Descartes and later by James (1890) describe emotions as epiphenomena, or non-functional feelings, derived from physiological changes or behaviour in response to provoking stimuli. Behaviourists, such as Skinner (1974) define emotions in terms of reinforced patterns of behaviour. However, ever since Aristotle, others have emphasised the importance of cognitions (thoughts, memories) as causal to emotions, with theorists such as Lyons (1992) describing the appraisal or interpretation of events, which then leads to physiological changes, as central to the formation of an emotion. An emotion could be defined as the value ascribed to events. Ekman (1992), has described emotions as "having evolved through their adaptive value in dealing with fundamental lifetasks". He argues that there are a finite number of emotions, each characterised by a distinctive facial expression, physiology, antecedents and presence in other primates.

How many different emotions are there? One theory (Lyons, 1992) argues against separate emotions, but instead suggests that a general level of arousal will be interpreted or 'coloured' by the individual in terms of the events and evaluations with which it is associated. Davidson (1992) has proposed a single emotion dimension built upon primitive adaptive responses: approach (positive) through to withdrawal (negative). The other type of theory (Darwin, 1872; Ekman, 1992) argues for the existence of separate, basic emotions, proposing six: sadness, happiness, anger, surprise, fear and disgust. In psychiatry, fear and sadness have been considered important as underlying several disorders, including specific and generalised anxiety and obsessive-compulsive disorder (OCD), and affective disorders, respectively. Evidence for abnormal perception of disgust as important in the genesis of specific psychiatric conditions is beginning to emerge and will be discussed below. We will firstly describe the defining characteristics of this otherwise neglected basic emotion.

\section{DISGUST}

Disgust (literally - bad taste) has been defined in terms of a food-related emotion. Darwin (1872) wrote that disgust was ". . . something offensive to the taste", and later authors (Rozin \& Fallon, 1987) describe the emotion as "revulsion at the prospect of (oral) incorporation of an offensive object'. The prototypical objects of disgust have been identified as waste products of the human and animal body but extend to other biological substances such as blood, saliva, sweat and hair. In addition, the concept of disgust can be expanded to involve violation of body borders at points other than the mouth (Rozin \& Fallon, 1987). This concept of core disgust can be further elaborated to include: animal-origin disgust, with the tendency of humans to emphasise the human-animal boundary (Rozin \& Fallon, 1987); interpersonal contamination, with disgust elicited by physical contact with unpleasant or unknown people (Rozin et al, 1994); and, finally, the moral or socio-cultural domain of the emotion (Miller, 1997), with disgust at certain beliefs or behaviours, such as sexual abuse of children, acting as a powerful means of transmitting social values (Rozin \& Fallon, 1987).
What constitutes a disgusting stimulus, at the margins of the definition, is clearly influenced by culture and individual differences. Such influences may be enshrined in religious dietary laws and national dietary preferences (consider the French taste for snails, horsemeat and steak tartar and British preference for black pudding and tripe). Lack of tolerance for sweat and body odour seem to be a recent concern in Western cultures. It has also been argued that complex emotions, such as shame, guilt and embarrassment, are derived from the basic emotion of disgust, with the focus being on the self (Power \& Dalgleish, 1997). Any emotion could be derived from combinations of basic emotions in the same way that an infinite number of hues can be derived from three basic colours.

\section{Psychoanalytic theories}

Freud (1908) considered disgust to be a reaction formation, that is a defence mechanism involving the repudiation of the desired object. This could become elaborated according to the developmental context so as to facilitate weaning, toilet training and sexual restraint. Modern versions of such theories stress disgust as a reaction against threats to the integrity of the self and as a means of imposing distance between self and object (Miller, 1993).

\section{The biology of disgust}

The reaction of a person to a disgusting object includes a characteristic facial expression (Rozin \& Fallon, 1987), with narrowing of the nostrils and raising of the upper lip (activity on the levator labii muscles), in addition to wrinkling of the brow (activity in the corrugator region). The purpose of the facial expression is to inhibit oral ingestion and includes the accompanying nausea and excessive salivation, but may extend to vocalisations ('yuk!', 'ugh!') and the shudder response.

Although non-human species do not demonstrate disgust, most avoid decaying meat, suggesting that they have an analogous form of food rejection. Perception of disgust in humans is absent at birth, with young children appearing to be tolerant of odours associated with decay, and children in the first two years of life demonstrating the well known tendency to put anything into their mouths. The disgust facial expression can, however, be elicited in young children in response to unpleasant tastes. Rozin \& Fallon (1987) concluded that 
perception of disgust develops at a later stage in childhood, between the ages of two and three and further at seven to nine years, probably as a result of cognitive development leading to ideational rejection of initially distasteful stimuli.

\section{The neurology of emotion perception}

There has been much recent interest in the investigation of the neural correlates of perception of positive and negative emotion. Lesion studies have implicated both the left hemisphere (Young et al, 1993) and right hemisphere (Adolphs et al, 1996) in the perception of facial expressions per se. The right hemisphere, however, has been demonstrated to have a role in the perception of negative emotions, in particular, sadness and fear (Adolphs et al, 1996; Gur et al, 1994).

There have been several studies investigating the specific neural substrate for perception of fear, with the amygdala emerging as critical to the perception of fearful facial expressions (Adolphs et al, 1994; Morris et al, 1996). However, there has been little investigation of the neural correlates of perception of disgust. Recent studies have demonstrated impaired recognition of disgust in people with Huntington's disease (Sprengelmeyer et al, 1996). This suggests that perception of disgust may involve palaeocortical structures atrophied in Huntington's disease, such as periamygdalar and piriform cortex. Other important structures are those implicated in appreciation of offensive stimuli, including a specific cortico-striatal-thalamic circuit identified in primates (Alexander et al, 1990). Evidence for the involvement of this circuit in perception of facial expressions of disgust, in addition to involvement of the anterior insula, identified as gustatory cortex in primates (Rolls et al, 1994) has been found in a recent functional imaging study (Phillips et al, 1997). The authors concluded that the neural response to facial expressions of disgust in others is thus closely related to appraisal of distasteful stimuli.

\section{DISGUST AS AN EMOTION UNDERLYING PSYCHIATRIC DISORDERS}

There are several lines of evidence to suggest that abnormal perception of disgust may underlie various psychiatric disorders, including OCD, phobias, depression, eating disorders, certain sexual dysfunctions and uncommon psychiatric syndromes.

\section{Obsessive-compulsive disorder}

There is accumulating evidence for the nonhomogeneity of OCD from study of the onset, symptom profile, treatment and underlying brain dysfunction (Blanes \& McGuire, 1997). There is a subgroup of people with $O C D$, with a chronic, deteriorating course, earlier age of onset and more severe symptoms. This subgroup is more common in males and can be linked with basal ganglia dysfunction. A second subtype, which is more in fermales, is associated with a later onset, mood and anxiety disorders, frontal lobe dysfunction and a good response to serotonergic antidepressants. One question is whether these two subgroups can be distinguished on the basis of perception of disgust, since a prominent theme of obsessional thoughts concern dirt/ contamination and washing, or checking. The cleaning/washing obsessions occur more often in females with a later age of onset of $O C D$, suggesting the relevance of disgust to this group in particular.

Perhaps more compelling evidence in favour of disgust as an emotion underlying OCD is demonstrated by the results of the functional neuroimaging studies, and the relationship of these with the findings from work investigating the neural correlates of perception of disgust. Reviews have highlighted increased metabolism, blood flow in orbitofrontal and striatal regions (Breiter \& Rauch, 1996). Increased activation in similar brain regions has been demonstrated during perception of facial expressions of disgust (Phillips et al, 1997).

\section{Phobias}

In their recent review, Power \& Dalgleish (1997) discuss evidence suggestive of a role for disgust in the genesis of certain phobias. Certain animal phobias may be disgustbased reactions to contamination or association with particular animal products such as mucus and faeces. Furthermore, they suggest that social phobia, in which the person imagines that he will be humiliated in public, may also have as its basic emotion self-directed disgust, or shame, a complex emotion perhaps related to disgust, as described above. Finally, they note that the physiological response to blood demonstrated by people with blood phobias (bradycardia and hypotension) is similar to that for perception of disgust, and very different from the usual phobia-induced fight-or-flight response (tachycardia).

\section{Depression}

The importance of shame in the development of self-esteem and relationship with others has been reviewed extensively (Gilbert, 1997). Low self-esteem is known to be an important component of depression, and self-loathing, or self-disgust, in addition to sadness, may represent part of the core phenomenological state of depression. Disgust would therefore appear to play an important role in the genesis of depression.

\section{Eating disorders}

People with anorexia nervosa and bulimia often experience disgust with respect to sexuality, parts of their bodies, and towards certain foods, especially those which are fattening or have a high calorie content. Those with bulimia nervosa appear to be able to overcome the normal response to satiety, and overeat, or 'binge', frequently enjoying the sensation of eating. However, the experience of self-directed disgust, characterised by feelings of being unattractive and overweight, after such binges (Russell, 1979) appears to contribute to the switch to the vomiting and purging part of the cycle. Individuals occasionally describe choosing repellent foodstuffs during a binge as an attempt to control the binge urge and perhaps as part of self-punishment.

It will be interesting in the future to investigate the perception of disgust, and the neural substrate underlying this, in people with eating disorders.

\section{Dysmorphophobia}

Dysmorphophobia, the persistent preoccupation with a presumed deformity or disfigurement and the refusal to accept advice and reassurance from others to the contrary, can also be linked with disgust (Thomas \& Goldberg, 1995).

\section{Other psychiatric disorders}

Coprophagia - usually defined as the ingestion of one's own faeces, is seen in the extremes of life in people with severe learning disabilities or dementia. Bilateral damage to the temporal lobes-amygdala complex is often implicated (Ghaziuddin \& MacDonald, 1985). 
Self-related disgust can be related to various sexual dysfunctions, including vaginismus, dyspareunia, premature ejaculation, erectile and orgasmic dysfunction (Power \& Dalgleish, 1997) and perversions. Uncommon psychiatric syndromes, such as lycanthropy, a delusion of metamorphosis into a wolf-like animal often in the context of sexual conflict, and vampirism, blood ingestion resulting in erotic satisfaction (Fahy et al, 1988), may also be associated with distorted perception of disgust. As for klismaphilia (the erotic enema deviance), urolagnia (ingestion of urine for sexual purposes), necrophilia/ phagia, bestiality (Rebal, 1982), one could speculate that th intense emotional response induced by disgusting stimuli and activities becomes associated with sexual arousal (Kraft-Ebing, 1886). Nevertheless, 'normal' eroticism from kissing onwards, presumably involves a similar but limited process, specific and personalised to the sexual partner.

\section{REFERENCES}

Adolphs, R., Tranel, D., Damasio, H., et of (1994) Impaired recognition of emotion in facial expressions following bilateral damage to the human amygdala. Nature, 372, 669-672.

_.. _. , ot ol (I996) Cortical systems for the recognition of emotion in facial expressions. Neuroscience. 315. 7678-7687.

Alexander, G. E., Crutcher, M. D. \& DeLong, M. R. (1990) Basal ganglia-thalamocortical circuits: parallel substrates for motor, oculomotor, "prefrontal" and "limbic" functions. Progress in Brain Reseorch, 85, 119-146.

Blanes, T. \& McGuire, P. K. (1997) Heterogeneity of obsessive-compulsive disorder: evidence for primary and neurodevelopmental subtypes. In Neurodevelopment in Psychiatry (eds R. Murray \& M. Keshevan). pp. 206212. Cambridge: Cambridge University Press.

Breiter, H. C. \& Rauch, S. L. (1996) Functional MR! and the study of OCD: from symptom provocation to cognitive-behavioural probes of cortico-striatal systems and the amygdala. Neuromoge, 4 (suppl). S127-S138.

M. L. PHILLIPS, MRCPsych, C. SENIOR, BSC. Department of Psychological Medicine, Institute of Psychiatry, London; T. FAHY, MRCPsych. Maudsley Hospital, London; A. S. DAVID. MD. Department of Psychological Medicine, Institute of Psychiatry, London

Correspondence: M. L. Phillips. Department of Psychological Medicine, King's College School of Medicine and Dentistry and Institute of Psychiatry, 103 Denmark Hill, London SES 8 AZ

(First received 12 August 1997, final revision 22 December 1997, accepted 22 December 1997)

Darwin, C. (1872) The Expression of the Emotions in Mon and Anmals. Reprinted 1965. Chicago, 1L: University of Chicago Press.

Davidion, R. J. (1992) Prolegomenon to the structure of emotion: gleanings from neuropsychology. Cognition and Emotion, 6. 245-268.

Ekman, P. (1992) An argument for basic emotions. Cognition and Emotion. 6. 169-200.

Fahy, T., Wessely, S. \& David, A. (1900) Werewolves. vampires and cannibals. Medicine. Science ond the Low, 28. $145-149$.

Freud, S. (1908) Character and anal eroticism. Reprinted (1953-1974) in The Stondard Edition of the Complete Psychological Works of Sigmund Freud (trans. and ed. J. Strachey), vol. 9. London: Hogarth Press.

Gharluddin, N. \& McDonald, C. (1985) A clinical study of adult coprophagics. British journal of Psychiotry, 147. $312-313$

Gilbert, P. (1997) The evolution of social attractiveness and its role in shame, humiliation. guilt and therapy. British journal of Medical Psychology, 70. 113-147.

Gur, R. C., Skolnick, B. E. \& Gur, R. E. (1994) Effects of emotional discrimination tasks on cerebral blood flow: regional activation and its relation to performance. Brain and Cognition, 25, 271-286.

James, W. (1890) The Principles of Psychology (vols I and 2). New York: Holt

Kraft-Ebing, R. Von (1886) Psychopathio Sexualis (trans. F. S. Klaft), published 1965. New York: Stein and Day.

Lyons, $w$. (1992) An introduction to the philosophy of emotions. In International Revew of Studies on Emotion (ed. K. T. Strongman), vol. 2. Chichester: Wiley.

Milier, S. B. (1993) Disgust reactions: their determinants and manifestations in treatment. Contemporary Psychoonalysis, 29, 711-735.

- (1997) The Anatomy of Disgust. Cambridge, MA: Harvard University Press.
Morris, J. S., Frith, C. D., Perrett, D. 1., ot ol (1996) A differential neural response in the human amygdala to fearful and happy facial expressions. Noture, 381. $812-815$.

Phillips, M. L., Young, A. W., Senior, C., et of (1997) A specific neural substrate for perception of facial expressions of disgust. Noture, 389, 495-498.

Power, M. \& Dalzleish, T. (1997) Cognition and Emotion From Order to Disorder. Hove: Psychology Press, Ertbaum. Taylor and Francis.

Rebal, R. F. (1992) Unusual sexual syndromes. In Extroordinary Disorders of Human Behovior (eds C. T. H. Friedman \& R. A. Faguet), pp. 121-154. New York: Plenum Press.

Rolls, E. T. (1994) Central Taste Anatomy and Physiology. In Handbook of Clinical Olfoction and Gustation (ed. R. L. Doty). New York: Dekker.

Rozin, P. \& Fallon, A. E. (1987) A perspective on disgust. Psychologicol Review, 94, 23-4!.

—, Lowery, L. Ebert, R. J. (1994) Varieties of disgust faces and the structure of disgust. journol of Personolity and Sociol Psychology, 66. 870-881

Russell, G. (1979) Bulimia nervosa: an ominous variant of anorexia nervosa. Psychological Medicine, 9, 429-448.

Skinner, B. F. (1974) About Behaviourism. New York: Alfred A. Knopf.

Sprengelmoyer, R., Young, A. W., Calder, A. J., ot ol (1996) Perception of faces and emotions: loss of disgust in Huntington's disease. Broin, 119. 1647-1665.

Thomas, C. S. \& Goldbers, D. P. (1995) Appearance, body image and distress in facial dysmorphophobia. Acto Psychiatrica Scondinavica, 92, 231-236.

Young, A. W., Nowcombe, F., de Haan, E. H. F., ot af (1993) Face perception after brain injury. Selective impairments affecting identity and expression. Brain, 116. 941-959. 